\title{
ESTUDO COMPARATIVO DE DIFERENTES METODOLOGIAS NA DETERMINAÇÃO DA EVAPOTRANSPIRAÇÃO DE REFERÊNCIA EM FEIRA DE SANTANA - BA
}

\author{
SOUZA, Tailan Santos de - tailan_santoss@hotmail.com \\ Universidade Estadual de Feira de Santana - UEFS
}
EICHENBERGER, Andréa Marina Rosário - marina.eichenberger@gmail.com
Universidade Estadual de Feira de Santana - UEFS

NASCIMENTO, Patrícia dos Santos - patysnasc@gmail.com
Universidade Estadual de Feira de Santana - UEFS

\begin{abstract}
RESUMO: Diferentes metodologias estimam a evapotranspiração e identificam qual método se adapta melhor a uma determinada região, auxiliando no processo de gerenciamento dos recursos hídricos. O objetivo do trabalho foi avaliar, dentre seis metodologias (Blaney-Criddle, Camargo, Hamon, Hargreaves-Samani, Linacre e Thornthwaite), a que melhor se aproxima da equação de referência (Penman-Monteith), em relação aos valores médios diários da evapotranspiração do município de Feira de Santana - BA. Foram calculadas as evapotranspirações para cada equação empírica em relação ao período de 2010 a 2014, seguidas de uma análise comparativa através do índice de concordância (d), índice de confiança (c), índice de correlação de Pearson ( $r$ ), regressão linear e das médias diárias mensais. Dos resultados encontrados, destaca-se o método de Camargo com $\mathrm{d}=0,69$ e $\mathrm{r}=0,72$, além de apresentar uma regressão linear mais próxima a equação de Penman-Monteith. Hargreaves-Samani se destaca com $c=$ 0,78 e o método de Blaney-Criddle foi o mais expressivo em relação a análise das médias diárias para os meses do ano. A equação de Camargo apresentou maior recorrência entre as análises comparativas, apresentando um desempenho mais próximo da equação de referência.
\end{abstract}

Palavras-Chave: Correlação; Coeficiente de desempenho; Gestão hídrica; PenmanMonteith

ESTUDIO COMPARATIVO DE DIFERENTES METODOLOGÍAS EN LA DETERMINACIÓN DE LA EVAPOTRANSPIRACIÓN DE REFERENCIA EN FEIRA DE SANTANA - BA

RESUMEN: Diferentes metodologías estiman la evapotranspiración e identifican qué método se adapta mejor a una región determinada, ayudando en el proceso de gestión de los recursos hídricos. El objetivo del trabajo fue evaluar, entre seis metodologías (Blaney-Criddle, Camargo, Hamon, Hargreaves-Samani, Linacre y Thornthwaite), que acerca mejor de la ecuación de referencia (Penman-Monteith), en relación con los valores promedio diarios de la evapotranspiración del municipio de Feira de Santana - BA. Fueron calculadas las evapotranspiraciones para cada ecuación empírica en relación el período 2010-2014, seguidas de un análisis comparativo a través de índice de acuerdo (d), el índice de confianza (c), el índice de correlación de Pearson (r), la regresión lineal y de promedios diarios mensuales. De los resultados encontrados, el método de Camargo se destaca con $d=0.69$ y $r=0.72$, además de presentar una regresión lineal más cercana a la ecuación de base. Hargreaves-Samani destaca con $c=0.78$ y el método de BlaneyCriddle fue el más expresivo en relación con el análisis de promedios diarios para los meses del año. La ecuación de Camargo presentó una mayor recurrencia entre los análisis comparativos, presentando un rendimiento más cercano a la ecuación de referencia.

Palabras Clave: Correlación; Coeficiente de rendimiento; Gestión del agua; PenmanMonteith 
COMPARATIVE STUDY OF DIFFERENT METHODOLOGIES IN THE DETERMINATION OF EVAPOTRANSPIRATION OF REFERENCE IN FEIRA DE SANTANA - BA

ABSTRACT: Different methodologies estimate evapotranspiration and identify which method adapts best a given region, assisting in the water resource management process. The objective of this work was to evaluate, among six methodologies (Blaney-Criddle, Camargo, Hamon, Hargreaves-Samani, Linacre and Thornthwaite), to what best approximates the reference equation (Penman-Monteith), in relation to the daily mean values of the evapotranspiration of the municipality of Feira de Santana - BA. Were calculated the evapotranspirations for each empirical equation in relation to period 20102014, followed by a comparative analysis through agreement index (d), confidence index (c), Pearson correlation index ( $r$ ), linear regression and of monthly daily averages. From the results found, we highlight the Camargo method with $d=0,69$ and $r=0,72$, besides presenting a linear regression closer to the base equation. Hargreaves-Samani excels with $\mathrm{c}=0,78$ and the Blaney-Criddle method was the most expressive in relation to the analysis of the daily averages for the months of the year. The Camargo equation presented higher recurrence among comparative analyzes, presenting a performance closer to the reference equation.

Keywords: Correlation; Coefficient of performance; Water management; PenmanMonteith.

\section{INTRODUÇÃO}

Segundo Pokorny (2019), a evapotranspiração é definida como a perda de água dos solos, corpos hídricos e superfícies vegetativas para a atmosfera através da vaporização da água líquida e é constituída pela soma de dois fenômenos, a transpiração e a evaporação. A transpiração é caracterizada pela água absorvida pela planta, através das raízes e liberadas pelos seus estômatos nas folhas, como medida de regularização de temperatura. Já a evaporação é a água que evapora das superfícies do solo, das folhas das plantas e aquáticas.

Nesse sentido, estimar a evapotranspiração de uma localidade ou cultura pode ser um fator importante para atividades de irrigação, gerenciamento de reservatórios, planejamento de uso e outorga dos recursos hídricos, bem como, para determinação do balanço hídrico (BORGES; MENDIONDO, 2007). O Instituto da Água (2001) afirma que a disponibilidade de dados precisos e confiáveis de evapotranspiração das culturas poderia alcançar uma economia média de até $20 \%$ da água utilizada para a irrigação no Brasil, evidenciando a sua relevância para o sistema hidrológico.

A determinação da evapotranspiração é feita através da Evapotranspiração de Referência (ETo), definida como o processo de perda de água para a atmosfera por meio de uma superfície padrão gramada, cobrindo a superfície do solo e sem restrição de umidade. Para a sua estimativa, a Food and Agriculture Organization (FAO) considera o método Penman-Monteith como método padrão (ALLEN et al., 1998).

O método de Penman-Monteith exige dados de medições de vários elementos climáticos como: temperatura do ar, umidade relativa, radiação solar e velocidade do vento. Assim, o uso de diversas variáveis climatológicas e a dificuldade em se encontrar essas informações corroboram para o uso de equações empíricas mais simplificadas, que utilizem menos variáveis climáticas, como os métodos de Thornthwaite (1948), Hargreaves-Samani (1985), BlaneyCriddle (1950), Camargo (1971), Hamon (1961) e Kharrufa (1985) (CONCEIÇÃO; MANDELLI, 2005). 
Ao se trabalhar com um banco de dados referentes à elementos climáticos, nota-se a necessidade de preenchimento de falhas nas séries temporais. Existe uma gama de técnicas geoestatísticas que possibilitam o preenchimento de lacunas existentes em uma variável distribuída no tempo (GOMES et al., 2019). O método da krigagem tem sido o mais indicado para análises de distribuição temporal de variáveis climatológicas (FERREIRA FILHO et al., 2019).

Devido ao grande número de métodos existentes para estimativa da ETo, a escolha do método mais adequado depende da disponibilidade de dados climatológicos, do nível de precisão exigido, da finalidade, dos custos e recursos financeiros disponíveis (TAGLIAFERRE et al., 2010).

Portanto, teve-se como objetivo avaliar quais dos métodos (BlaneyCriddle (1950), Camargo (1971), Hamon (1961), Hargreaves-Samani (1985), Linacre (1977) e Thornthwaite (1948)) melhor se aproxima da metodologia de referência (Penman-Monteith), em relação aos valores médios diários da evapotranspiração do município de Feira de Santana-BA para o período de 2010 a 2014 através de diferentes análises comparativas.

\section{MATERIAL E MÉTODOS}

\section{ÁREA DE ESTUDO}

Feira de Santana é a segunda maior cidade da Bahia, com uma população estimada de 609.913 habitantes no ano de 2018, segundo o Instituto Brasileiro de Geografia e Estatística (IBGE, 2019). O município está situado no agreste baiano, com coordenadas geográficas (120 15' $24^{\prime \prime} \mathrm{S}$ e $37^{\circ} 57^{\prime} 53^{\prime \prime} \mathrm{W}$ ), em uma faixa entre a zona da mata e o sertão baiano, situando-se numa zona intermediária entre o clima úmido do litoral e o semiárido do interior (DINIZ et al., 2008). Segundo a tipologia de Köppen, o clima da região é do tipo "Asa", megatérmico com chuvas de inverno e verão quente (SANTOS et al., 2017). O município apresenta precipitação média anual de $848 \mathrm{~mm}$ e temperatura média anual de $24^{\circ} \mathrm{C}$, podendo atingir no verão médias mensais de $27^{\circ} \mathrm{C}$ e no inverno de $21^{\circ} \mathrm{C}$ (DINIZ et al., 2008). A amplitude térmica anual caracteriza-se em torno de $10^{\circ} \mathrm{C}$ e a amplitude diária fica em torno de $7^{\circ} \mathrm{C}$ no verão e $12^{\circ} \mathrm{C}$ no inverno, em média (SANTOS; ANDRADE, 2008). A Figura 01 apresenta a localização geográfica do município de Feira de Santana. 


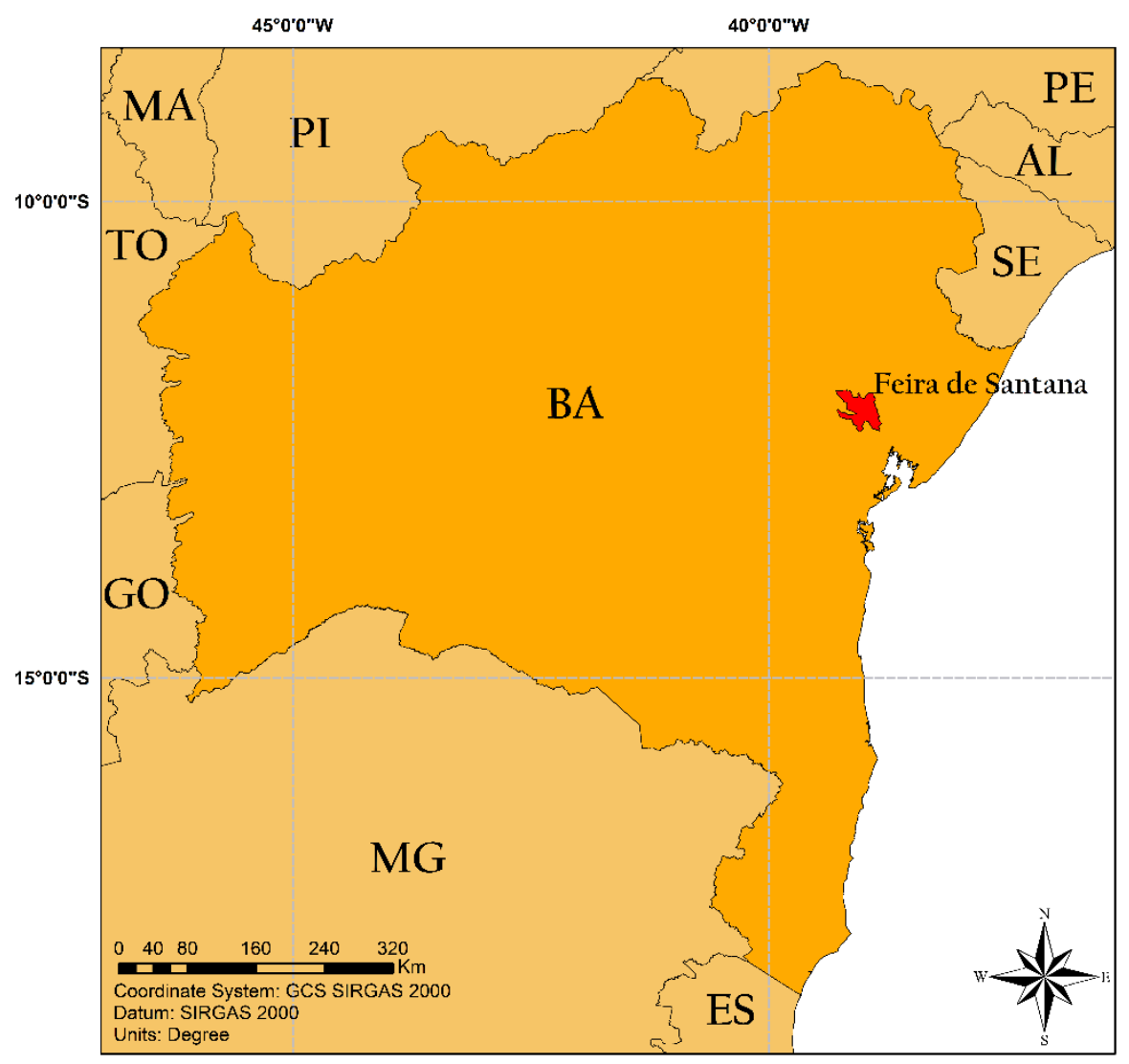

Figura 01 - Localização do município de Feira de Santana. Fonte: Autores, 2019.

\section{DADOS COLETADOS}

Os dados meteorológicos necessários para o cálculo da evapotranspiração foram obtidos no Banco de Dados de Recursos Hídricos (BDRH) e disponibilizados pela estação climatológica convencional de coordenadas geográficas $12^{\circ} 15^{\prime} \mathrm{S}$ e $38^{\circ} 5^{\prime} \mathrm{W}$, pertencente ao IV Distrito do Instituto Nacional de Meteorologia (INMET), localizada no Campus da Universidade Estadual de Feira de Santana. Os referidos dados compreendem as séries históricas diárias e mensais da insolação total (horas), da temperatura $\left({ }^{\circ} \mathrm{C}\right)$, da velocidade do vento $(\mathrm{m} / \mathrm{s})$ e do percentual de umidade relativa (\%). Para esse estudo, adotou-se o período entre os anos de 2010 e 2014, por ser o intervalo de tempo disponível onde os dados contêm menor número de falhas na série temporal.

Para o preenchimento das falhas, optou-se pelo método de interpolação da krigagem, que é um dos principais métodos de interpolação defendidos na literatura, já que ele estima o valor máximo da correlação espacial do parâmetro a ser estimado a partir da função do semivariograma (LANDIM, 2000; DEUS et al., 2010). As séries mensais e diárias com dados faltantes foram analisadas por meio do software Surfer, versão 9, da "Golden Software, Inc.", 2010, que executa a krigagem, gerando um mapa de interpolação temporal, onde estão indicados os meses e anos (para dados mensais) e os dias e meses (para dados 
diários) do período de estudo. Tais mapas geram as estimativas dos parâmetros faltantes do mês ou do dia que possua a lacuna na série histórica trabalhada.

Levando-se em consideração a influência dos períodos anuais, secos e chuvosos, na evapotranspiração de referência, foi obtida, também no banco de dados do INMET, a série histórica das precipitações mensais dentro do intervalo de tempo analisado. Com a precipitação média mensal do período, foi construído o histograma de chuvas da Figura 02, que apresenta os meses de abril a julho como chuvosos.

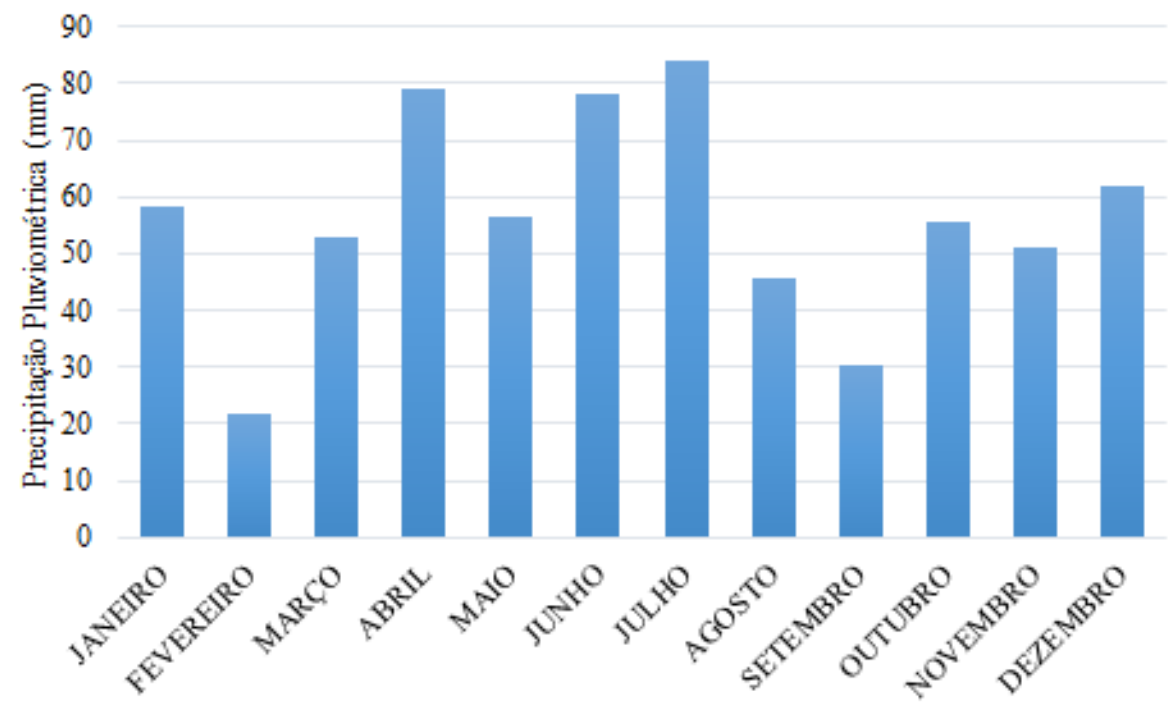

Figura 02 - Histograma de precipitação do município de Feira de Santana (de 2010 a 2014). Fonte: Autores, 2019.

Para cada ano do período em estudo, foram avaliados e comparados os valores médios mensais da estimativa da evapotranspiração de referência, em $\mathrm{mm}$ por dia, utilizando-se dos seguintes métodos: Penman-Monteith (ALLEN et al., 1998), Thorntwaite (THORNTWAITE, 1948), Blaney-Criddle (DOORENBOS; PRUITT, 1977), Camargo (CAMARGO, 1971), Hargreaves-Samani (HARGREAVES; SAMANI, 1985), Linacre (LINACRE, 1977) e Hamon (HAMON, 1961).

\section{DESCRIÇÃO DOS MÉTODOS PARA ESTIMATIVA DA EVAPOTRANSPIRAÇÃO DE REFERÊNCIA}

MÉTODO DE PENMAN-MONTEITH

Foi apresentado, no Relatório Técnico em Irrigação e Drenagem no 56 da Food and Agriculture Organization (FAO), como o método padrão para obtenção de valores diários de evapotranspiração de referência (ALLEN et al., 1998). Segundo Souza et al. (2015), esse método requer observações de radiação solar, temperatura, umidade do ar e velocidade do vento, informações obtidas através de estações meteorológicas, que não são tão recorrentes no Brasil e apresentam grandes lacunas nas séries históricas, o que acaba dificultando a aplicação dessa metodologia. 
Para o cálculo dessas variáveis, a metodologia desenvolvida inclui recomendações para situações de escassez de dados, como é o caso da densidade do fluxo de calor no solo (G), que pode ser considerada nula quando não existir valor observado. Para o saldo da radiação líquida $(R n)$, o cálculo foi efetuado somando-se as parcelas do saldo de radiação de ondas curtas (Rns) e do saldo de ondas longas ( $\mathrm{Rnl})$, que dependem basicamente dos dados de altitude, latitude, dia Juliano e pressão atmosférica (MEDEIROS, 2002). A Equação 01 descreve como são obtidos os valores de evapotranspiração de referência pelo método de Penman-Monteith.

$$
E T_{o}=\frac{0,408 * \Delta *\left(R_{n}-G\right)+\gamma * \frac{900}{T+273} * u_{2} *\left(e_{s}-e_{a}\right)}{\Delta+\gamma *\left(1+0,34 * u_{2}\right)}
$$

(Equação 01)

Nesta equação, $\Delta$ é a declinação da curva de saturação do vapor de água $\left(\mathrm{kPa} /{ }^{\circ} \mathrm{C}\right), \mathrm{Rn}$ é o saldo de radiação solar $\left(\mathrm{MJ} / \mathrm{m}^{2}\right.$.dia), G é a densidade do fluxo de calor no solo $\left(\mathrm{MJ} / \mathrm{m}^{2}\right.$.dia), y é a constante psicrométrica $(\mathrm{MJ} / \mathrm{kg})$, T é a temperatura média do ar $\left({ }^{\circ} \mathrm{C}\right)$, U2 é a velocidade do vento (média diária) a $2 \mathrm{~m}$ acima da superfície do solo $(\mathrm{m} / \mathrm{s})$, es é a pressão de saturação do vapor $(\mathrm{kPa})$ e ea é a pressão atual do vapor $(\mathrm{kPa})$.

\section{MÉTODO DE THORNTHWAITE}

Proposto por Thornthwaite (1948), para estimativa da Evapotranspiração Potencial (ETP) mensal, o conjunto de equações dessa metodologia utiliza somente a temperatura como variável independente e foi baseado no balanço hídrico e nas medidas de evapotranspiração realizadas em lisímetros. Camargo e Camargo (2000) constataram, no seu estudo, que o método apresenta resultados comparáveis e satisfatórios em regiões de clima úmido, ao mesmo tempo em que subestima a ETP para climas mais áridos. A Equação 02 descreve a obtenção da evapotranspiração potencial mensal corrigida pelo método de Thornthwaite.

$$
E T_{o}=E T P_{p} * \frac{N D}{30} * \frac{N}{12}
$$

(Equação 02)

Onde ND é o número de dias do mês, $\mathrm{N}$ é o fotoperíodo do $15^{\circ}$ dia do mês (horas) e ETPp é a evapotranspiração potencial padrão de Thornthwaite, dada pela Equação 03.

$$
\operatorname{ETP}_{p}=16 *\left(\frac{10 * \text { Tmed }}{I}\right)^{a}
$$

(Equação 03)

Para se calcular a evapotranspiração padrão, considera-se que: Tmed é a temperatura média em graus célsius; I é o índice de calor anual, que depende das temperaturas médias de cada mês; e a é um coeficiente baseado no índice de calor anual (THORNTHWAITE, 1948). 


\section{MÉTODO DE BLANEY-CRIDDLE}

Constitui uma das metodologias mais antigas para a determinação da evapotranspiração. Passada por algumas revisões, teve uma de suas versões apresentada pela FAO como método de cálculo para evapotranspiração de referência, sendo pouco indicado para regiões em que a temperatura do ar se mantenha estável (DOORENBOS; PRUITT, 1977). Este método estima a ETo média mensal ( $\mathrm{mm} / \mathrm{dia}$ ) de um gramado sendo obtida pela Equação 04.

$$
E T_{o}=(0,457 * T+8,13) * p
$$

(Equação 04)

Tem-se que $\mathrm{T}$ é a temperatura média do ar $\left({ }^{\circ} \mathrm{C}\right)$ e $\mathrm{p}$ é a porcentagem do total de fotoperíodo médio mensal sobre o total de fotoperíodo anual, que é tabelado, varia para cada mês do ano e depende basicamente da latitude da região de estudo.

\section{MÉTODO DE CAMARGO}

Baseado nos resultados do método de Thornthwaite (1948), o método de Camargo (1971) define sua equação de forma mais simples e com a mesma eficiência na estimação da evapotranspiração de referência. Segundo Santos et al. (2010), esse método apresenta as mesmas vantagens e restrições de Thornthwaite, com exceção da vantagem de não se utilizar apenas a temperatura média como variável independente, considerando também a radiação solar. A Equação 05 descreve a obtenção da evapotranspiração mensal pelo método de Camargo.

$$
E T_{o}=0,01 * R_{a} * T_{a} * K
$$

(Equação 05)

Onde $\mathrm{K}$ é o fator de ajuste de Camargo, que varia de acordo com a Temperatura média do ar ( $\mathrm{Ta}$ ), Ra é a radiação solar extraterrestre incidente acima da atmosfera no dia 15 de cada mês ( $\mathrm{mm} / \mathrm{dia})$.

\section{MÉTODO DE HARGREAVES-SAMANI}

Usando dados obtidos em um lisímetro na cidade de Davis, estado da Califórnia, cujo clima é o semiárido, Hargreaves e Samani (1985) propuseram o seu modelo para a estimativa de evapotranspiração de referência média mensal. O método se baseia na temperatura média do ar e na amplitude térmica, possuindo como vantagem a sua aplicabilidade em climas áridos e semiáridos, como no nordeste do Brasil (SANTOS et al., 2010). A fórmula do método Hargreaves-Samani é apresentada na Equação 06.

$$
E T_{o}=0,0023 * Q_{o} *\left(T_{\operatorname{má} x}-T_{\min }\right)^{0,5} *\left(17,8+T_{\text {med }}\right)
$$

\section{(Equação 06)}

Onde Tmed é a temperatura média do ar $\left({ }^{\circ} \mathrm{C}\right)$, Tmáx e Tmín são as temperaturas máxima e mínima do $\operatorname{ar}\left({ }^{\circ} \mathrm{C}\right)$, respectivamente e Qo é a radiação solar extraterrestre diária. 
Buscando uma simplificação do antigo modelo de Penman (1948), Linacre (1977) desenvolveu um modelo para estimar a evapotranspiração de referência média, utilizando como parâmetros de referência os dados de temperatura, umidade relativa do ar, latitude e altitude. Em seu estudo, Soares et al. (2018) concluíram que o método de Linacre apresenta uma sensibilidade alta às variações de temperatura do ar, sendo que pequenas mudanças desse parâmetro alteram significativamente os valores da evapotranspiração. A Equação 07 é a fórmula da estimativa da evapotranspiração de referência pelo método de Linacre.

$$
E T_{o}=\frac{500 *\left(\frac{T_{\text {med }}+0,006 * h}{100-\phi}\right)+15 *\left(T_{\text {med }}-T_{p o}\right)}{\left(80-T_{\text {med }}\right)}
$$

(Equação 07)

Considera-se que: Tmed é a temperatura média em graus célsius; h é a altitude local em metros; $\varphi$ é o módulo da latitude em graus; e Tpo é a temperatura média do ponto de orvalho em graus, que depende da pressão de vapor atual (ea, em $\mathrm{mmHg}$ ), que, por sua vez, depende do percentual da umidade relativa local (LINACRE, 1977).

\section{MÉTODO DE HAMON}

Esse modelo foi desenvolvido por Hamon (1961), sua fórmula inclui o efeito da insolação, incorporando a duração do dia, baseado na latitude e no mês analisado. A ETo média diária para os meses estudados é expressa pela Equação 08.

$$
E T_{o}=0,55 *\left(\frac{N}{12}\right)^{2} *\left(\frac{4,93 * \exp ^{\left(0,062 T_{m e ́ d}\right)}}{100}\right) * 25,4
$$

(Equação 08)

Onde Tmed é a temperatura média do $\operatorname{ar}\left({ }^{\circ} \mathrm{C}\right)$ e $\mathrm{N}$ é o fotoperíodo do $15^{\circ}$ dia do mês (horas), que é um dado tabelado, obtido através da latitude local.

\section{COMPARAÇÃO DAS EQUAÇõES}

A análise comparativa das equações foi realizada através do índice de concordância desenvolvido por Willmott (1981), utilizando-se de um coeficiente denominado concordância ou precisão (designado pela letra "d"), obtido com a Equação 09, que quantifica matematicamente a dispersão dos dados em relação ao método considerado padrão, nesse caso, o Método de Penman-Monteith.

$$
d=1-\left[\frac{\sum\left(P_{i}-O_{i}\right)^{2}}{\sum\left(\left|P_{i}-O\right|+\left|O_{i}-O\right|\right)^{2}}\right]
$$

(Equação 09)

Em que: Oi são os valores estimados de evapotranspiração de referência para o método Penman-Monteith; Pi representa os valores estimados para cada uma das demais equações analisadas; e O é a média dos valores estimados para Penman-Monteith.

Para a análise da confiabilidade de cada equação, considerou-se um índice de confiança (c) proposto por Camargo e Sentelhas (1997), que é 
resultado do produto do índice de precisão (d) e do índice de correlação de Pearson ( $r$ ) descrito pela Equação 10. Essa última variável é obtida através da raiz do coeficiente de determinação $\left(r^{2}\right)$, referente a equação da reta de cada método descrita graficamente.

$$
c=d \times r
$$

(Equação 10)

Os valores dos índices de correlação e confiança foram interpretados conforme Cunha et al. (2013b) e Camargo e Sentelhas (1997), evidenciados nas Tabelas 01 e 02, respectivamente.

Tabela 01 - Classificação dos valores do índice de correlação de Pearson (r).

\begin{tabular}{cc}
\hline Índice de correlação $(r)$ & Classificação \\
\hline 0,0 a 0,1 & Muito baixa \\
0,1 a 0,3 & Baixa \\
0,3 a 0,5 & Moderada \\
0,5 a 0,7 & Alta \\
0,7 a 0,9 & Muito alta \\
0,9 a 1,0 & Quase perfeita
\end{tabular}

Fonte: Cunha et al., 2013b.

Tabela 02 - Critérios de interpretação do índice de desempenho (c).

Fonte: Camargo e Sentelhas, 1997.

\begin{tabular}{cc}
\hline Índice de desempenho (c) & Classificação \\
\hline$>0,85$ & Ótimo \\
0,76 a 0,85 & Muito bom \\
0,66 a 0,75 & Bom \\
0,61 a 0,65 & Mediano \\
0,51 a 0,60 & Sofrível \\
0,41 a 0,50 & Mau \\
$<0,40$ & Péssimo \\
\hline
\end{tabular}

\section{RESULTADOS E DISCUSSÃO}

Ao analisar os índices de correlação e classificação entre as diferentes metodologias verifica-se que o índice de correlação ( $r$ ) para os métodos estudados em comparação com o método padrão apresenta uma boa classificação, variando da classe "Muito alta" até a "Quase perfeita", alcançada pelas equações de Camargo e Hamon (Tabela 03).

Apesar dessa boa correlação obtida no índice anterior, dentre os seis métodos avaliados apenas dois apresentaram um resultado razoável em relação ao método de Penman-Monteith segundo o índice de desempenho (c), que foram Camargo e Hargreaves-Samani, ambos obtiveram um desempenho classificado como "Bom". Os demais, com exceção de Linacre que obteve o pior desempenho, classificado com o índice "Mau", apresentaram uma classificação "Sofrível". 
O bom desempenho observado pelo método de Hargreaves-Samani pode ser explicado pelo fato da equação ter sido ajustada para uso em regiões semiáridas, como o município em estudo (PEREIRA et al., 1997). Já o método de Camargo, utiliza um ajuste de variável de acordo com as temperaturas médias, o que pode ter colaborado para o resultado encontrado. Moura et al. (2013) afirma que a introdução do fator de ajuste adequado, pode proporcionar uma boa representação do fator aerodinâmico (calor latente) resultando em estimativas mais confiáveis.

Tabela 03 - Coeficientes de exatidão (d), correlação ( $r$ ) e desempenho (c), para valores diários de evapotranspiração de referência calculada por diferentes métodos de estimativa comparados com o método padrão de Penman-Monteith no período de 2010 a 2014 do município de Feira de Santana-BA.

\begin{tabular}{cccccc}
\hline $\begin{array}{c}\text { Métodos de estimativa de } \\
\text { ETo }\end{array}$ & $\mathrm{d}$ & $\mathrm{r}$ & $\mathrm{c}$ & $\begin{array}{c}\text { Classificação Índice } \\
\text { de correlação }(r)\end{array}$ & $\begin{array}{c}\text { Classificação do } \\
\text { índice de } \\
\text { desempenho (c) }\end{array}$ \\
\hline Blaney-Criddle & 0,66 & 0,77 & 0,51 & Muito alta & Sofrível \\
Camargo & 0,72 & 0,96 & 0,69 & Quase perfeita & Bom \\
Hamon & 0,57 & 0,94 & 0,54 & Quase perfeita & Sofrível \\
Hargreaves-Samani & 0,78 & 0,83 & 0,65 & Muito alta & Bom \\
Linacre & 0,57 & 0,87 & 0,50 & Muito alta & Mau \\
Thornthwaite & 0,66 & 0,87 & 0,58 & Muito alta & Sofrível \\
\hline
\end{tabular}

Fonte: Autores, 2019.

Syperreck et al. (2008) encontraram bom ajuste e desempenho dos métodos Hargreaves-Samani e Camargo, quando comparados com o de Penman-Monteith na escala diária e ambos modelos dependem da temperatura para sua realização. Santos et al. (2010) afirmaram que métodos empíricos, como o de Camargo, estimam bem a ETo na escala mensal, por envolverem poucas variáveis. Segundo Soares et al. (2018) o método de Linacre apresenta uma sensibilidade alta às variações de temperatura do ar e, como Feira de Santana possui uma razoável amplitude térmica, o método não é o mais adequado para a localidade, justificando assim o seu distanciamento da equação de base.

De acordo com a estimativa dos valores médios diários na escala mensal da evapotranspiração de referência para o município de Feira de Santana, notase que o método de Penman-Monteith estimou uma ETo média de 5,23 mm/dia, enquanto que dentre os demais métodos analisados, o que mais se aproximou dessa estimativa foi 0 de Blaney-Criddle com 5,21 mm/dia, seguido de Hargreaves-Samani com 5,01 mm/dia. Apesar do método de Blaney-Criddle melhor se aproximar do resultado encontrado pelo método padrão, quando se compara suas médias mensais são encontradas variações de $1,5 \%$ a 33,2\%, observados nos meses de abril e julho respectivamente, enquanto que Hargreaves-Samani apresenta uma variação de 2,7 a 23,1\% para os mesmos meses. Assim, analisar a média dos dados pode não ser a melhor alternativa comparativa de metodologias, visto a falta de homogeneidade nos resultados encontrados (Tabela 04). 
Tabela 04 - Variação percentual entre as diferentes ETo's e a ETo de referência (Penman-Monteith).

\begin{tabular}{cccccccc}
\hline \multirow{2}{*}{ Mês } & $\begin{array}{c}\text { ETo Padrão } \\
(\mathrm{mm})\end{array}$ & \multicolumn{7}{c}{ Variação percentual da ETo (\%) } \\
\cline { 2 - 7 } & $\begin{array}{c}\text { Penman- } \\
\text { Monteith }\end{array}$ & $\begin{array}{c}\text { Blaney- } \\
\text { Criddle }\end{array}$ & Camargo & Hamon & $\begin{array}{c}\text { Hargreaves- } \\
\text { Samani }\end{array}$ & Linacre & Thornthwaite \\
\hline Janeiro & 6,59 & $-12,0$ & $-22,6$ & $-39,6$ & $-13,2$ & $-37,2$ & $-28,2$ \\
Fevereiro & 6,47 & $-23,5$ & $-22,6$ & $-40,0$ & $-20,7$ & $-36,2$ & $-27,7$ \\
Março & 6,18 & $-9,1$ & $-21,4$ & $-39,0$ & $-7,3$ & $-32,4$ & $-20,4$ \\
Abril & 5,21 & $-1,5$ & $-23,0$ & $-37,0$ & $-2,7$ & $-28,2$ & $-19,0$ \\
Maio & 4,12 & $+26,0$ & $-24,3$ & $-29,9$ & $+11,7$ & $-19,7$ & $-14,8$ \\
Junho & 3,58 & $+30,4$ & $-27,4$ & $-28,5$ & $+18,7$ & $-15,6$ & $-19,3$ \\
Julho & 3,55 & $+33,2$ & $-27,0$ & $-30,1$ & $+23,1$ & $-20,3$ & $-26,2$ \\
Agosto & 4,08 & $+18,9$ & $-29,9$ & $-37,5$ & $+13,2$ & $-29,4$ & $-37,0$ \\
Setembro & 5,00 & $-2,2$ & $-32,8$ & $-42,8$ & $-5,2$ & $-35,6$ & $-40,4$ \\
Outubro & 5,64 & $-5,0$ & $-27,8$ & $-43,6$ & $-6,9$ & $-39,9$ & $-35,5$ \\
Novembro & 5,98 & $-7,9$ & $-23,7$ & $-37,8$ & $-14,0$ & $-39,3$ & $-31,3$ \\
Dezembro & 6,34 & $-8,7$ & $-24,3$ & $-37,9$ & $-12,3$ & $-39,1$ & $-28,9$ \\
Média & 5,23 & $-0,4$ & $-25,2$ & $-37,7$ & $-4,2$ & $-32,5$ & $-27,7$ \\
\hline
\end{tabular}

Fonte: Autores, 2019.

Hamon e Linacre foram os métodos que mais se afastaram do resultado encontrado em relação ao método padrão. Tais métodos se distanciaram em relação à média 37,7 e 32,5\% respectivamente. Esse distanciamento observado em Linacre, pode ser atribuído ao fato da metodologia não considerar a radiação e a velocidade do vento, resultado semelhante ao encontrado por Pereira et al. (2009) e Mendonça e Dantas (2010). Segundo Cunha et al. (2013a), apesar do método de Hamon apresentar a complexidade de abordar muitos coeficientes, sua metodologia necessita apenas de um parâmetro medido, que é a temperatura média do ar, diferentemente da metodologia padrão, que aborda diversas variáveis. Devido a essa dependência a uma única variável, a metodologia pode apresentar resultados não tão satisfatórios, como observado também em Borges e Mediondo (2007), Pereira et al. (2009) e Cavalcante Jr. et al. (2011).

A Figura 03 apresenta a variação média mensal da evapotranspiração de referência calculados pelos diferentes métodos aplicados no município de Feira de Santana no período de 2010 a 2014. A partir dela, pode-se observar que a curva que mais se aproxima do método padrão é a do modelo de HargreavesSamani.

Os menores valores observados para a evapotranspiração ocorreram entre maio e agosto, período que coincide com a maior parte dos meses do período chuvoso (abril até julho), no município de Feira de Santana. Esses valores baixos se devem, portanto, a menor disponibilidade de energia no sistema solo-planta-atmosfera para o fluxo de calor latente, neste período do ano. Resultados semelhantes foram encontrados por Araújo et al. (2007), Syperreck et al. (2008) e Moura et al. (2013). 


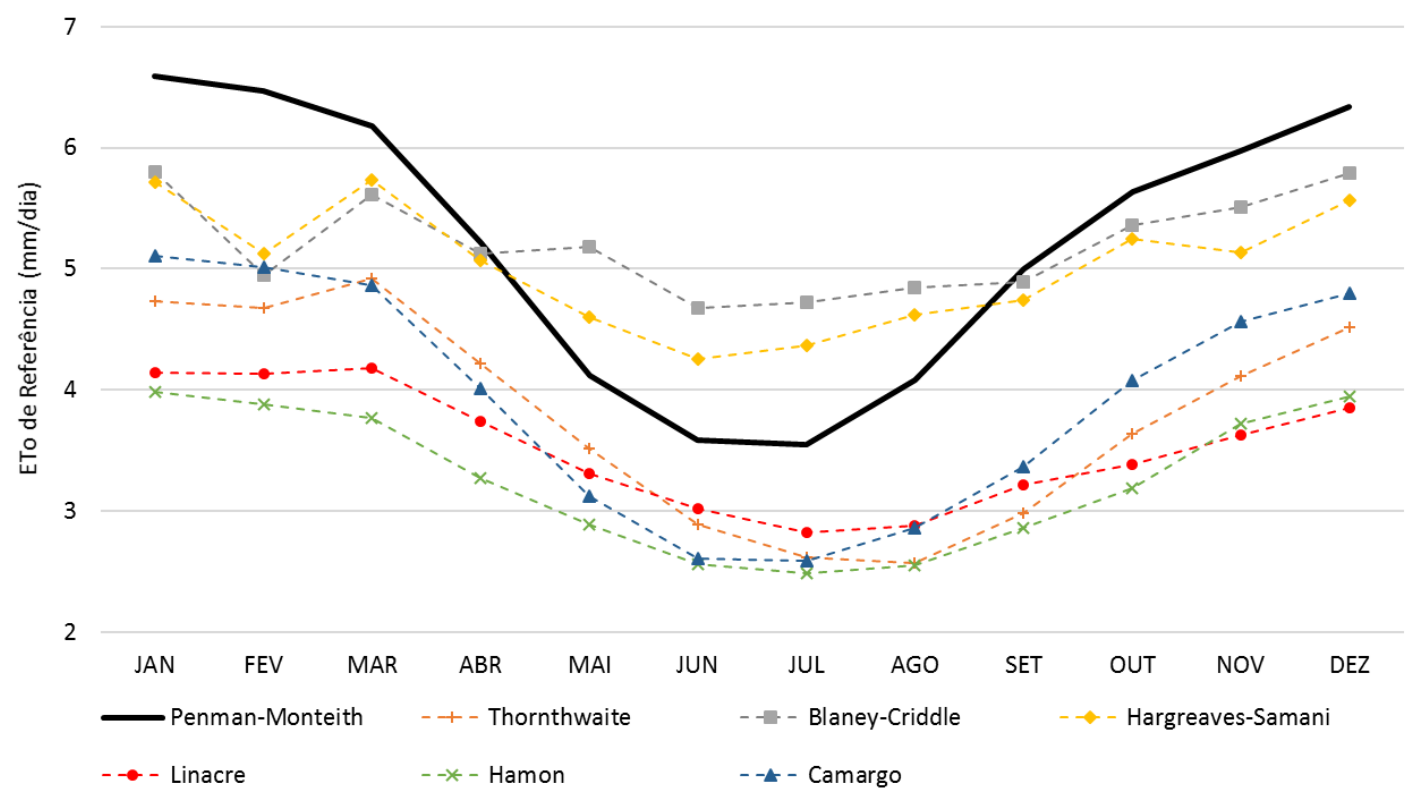

Figura 03 - Variação média mensal da Evapotranspiração de Referência. Fonte: Autores, 2019.

Foi possível observar que nos períodos relativamente mais secos (setembro a março), o método padrão de Penman-Monteith superestima a evapotranspiração em relação aos outros métodos avaliados. Já no período de maior pluviosidade (abril a agosto), apenas os métodos de Blaney-Criddle e Hargreaves-Samani superestimaram o método padrão, fato que também foi observado em ambos métodos por Araújo et al. (2007), apenas em BlaneyCriddle por Moura et al. (2013) e apenas em Hargreaves-Samani por Aragão et al. (2017).

Outra influência observada foi que, no período chuvoso, os métodos de Linacre, Hamon, Thornthwaite e Camargo tendem a resultar em estimativas mais próximas, o que não ocorre nos meses mais secos. Em relação a esses quatro métodos, cabe observar também, que, em termos médios de evapotranspiração mensal para o período de estudo, todos eles obtiveram uma estimativa abaixo do modelo padrão de Penman-Monteith. Primo et al. (2019) examinaram o mesmo comportamento para os métodos de Camargo e Thornthwaite, sendo que este último também foi percebido por Moura et al. (2013).

Na Figura 04 observa-se os gráficos de regressão linear da média diária mensal da evapotranspiração de referência calculados pelos diferentes métodos estudados. O coeficiente angular para as seis metodologias de estimativa de ETo são maiores que " 1 " indicando a tendência de superestimativa em relação à ETo obtida pelo método padrão. Enquanto que o coeficiente de determinação $\left(R^{2}\right)$ teve uma variação de aproximadamente 0,60 em Blaney-Criddle (Figura 04a) a 0,92 em Camargo (Figura 04b). Nota-se também que mesmo a metodologia de Linacre apresentando um coeficiente de determinação razoável de 0,75 (Figura 04e), sua avaliação quanto ao coeficiente de desempenho (c) foi a pior das metodologias. 
De forma gráfica, os resultados se apresentam bastantes dispersos em relação às retas $1: 1$, indicando seu distanciamento do método padrão proposto, com exceção do método de Camargo (Figura 04b), que é a equação que mais se alinha a essa reta, fato que pode ser observado também através do seu coeficiente de determinação, o mais próximo de 1. Cunha et al. (2013a) encontraram um resultado diferente, no qual o método de Camargo não apresentou certo alinhamento, que pode ser explicado devido as variabilidades de clima das diferentes cidades. 

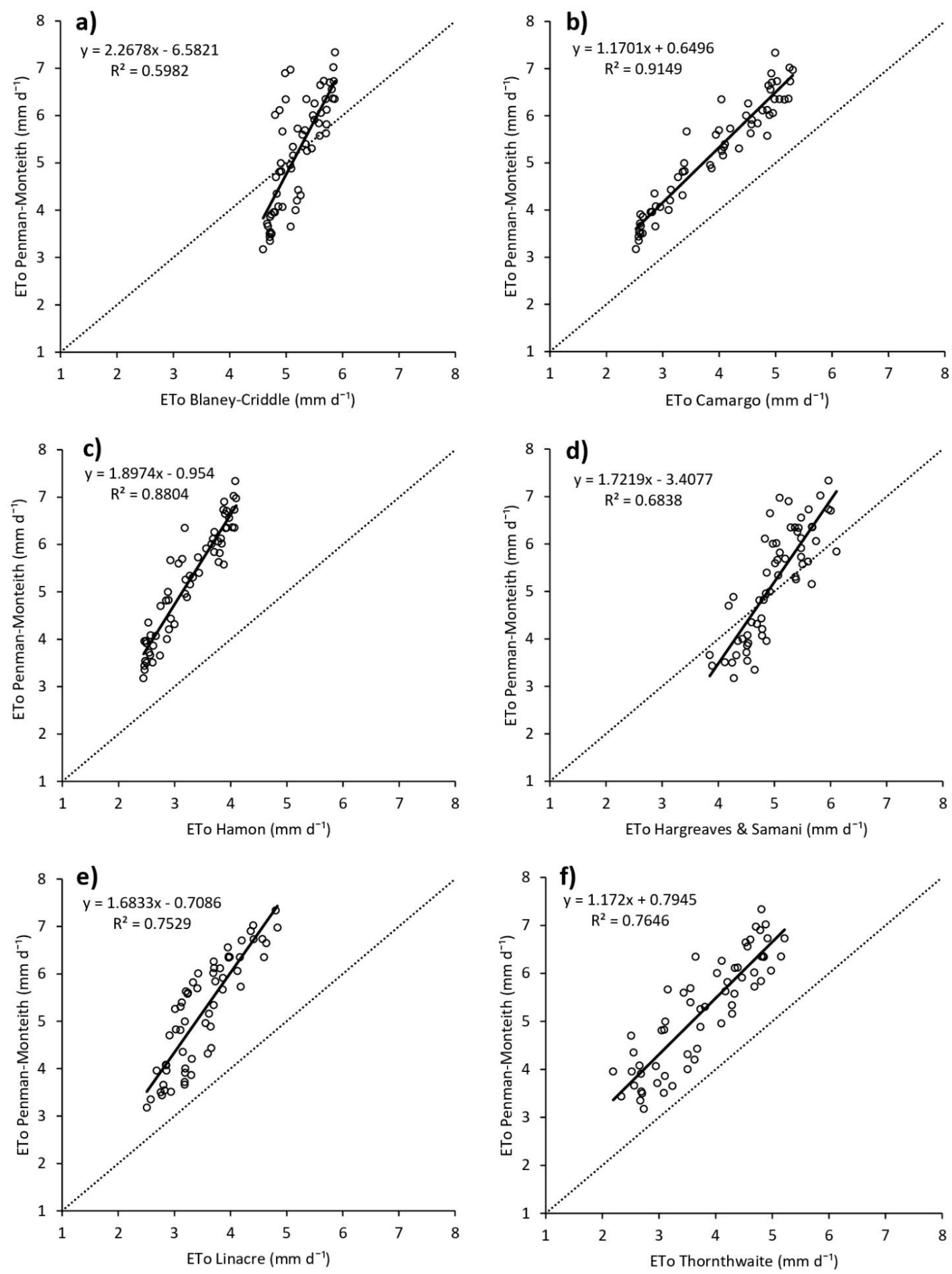

Figura 04 - Valores mensais de médias diárias de ETo (Penman-Monteith versus BlaneyCriddle (a), Camargo (b), Hamon (c), Hargreaves-Samani (d), Linacre (e) e Thornthwaite (f)) comparados por regressão linear para a cidade de Feira de Santana-BA, período 2010-2014. A linha pontilhada indica a reta 1:1. Fonte: Autores, 2019. 


\section{CONCLUSÕES}

De acordo com o índice de concordância (d) o método que mais se alinha ao de Penman-Monteith é o de Camargo, com $d=0,69$, classificado como "Bom". Já o índice de confiança (c), é melhor representado pelo de HargreavesSamani, com $c=0,78$ e o índice de correlação de Pearson possui melhor associação com o de Camargo, onde $r=0,72$.

A análise das médias diárias para os meses do ano no período de estudo indica o método de Blaney-Criddle o mais próximo do método padrão. Segundo a análise gráfica das médias diárias para cada mês do período, o modelo de Hargreaves-Samani é o que mais se aproxima do de referência. A regressão linear aponta o método de Camargo como o mais próximo ao de PenmanMonteith para as condições experimentais.

Dentre todos os parâmetros avaliados o método de Camargo foi o que apresentou maior proximidade a equação de referência proposta por PenmanMonteith.

\section{REFERÊNCIAS BIBLIOGRÁFICAS}

ALLEN, R. G.; PEREIRA, S. L.; RAES, D.; SMITH, M. Crop evapotranspiration: guidelines for computing crop water requirements. FAO - Irrigation and Drainage, 1998. 300 p.

ARAGÃO, F. T. de. A. et al. Comparação de três métodos de estimativa da evapotranspiração de referência para a região de Tauá - CE. In: JORNADA DE PÓS-GRADUAÇÃO E PESQUISA, 14., Bagé. Anais... Revista da Jornada de PósGraduação e Pesquisa Congrega, 2017. 9 p.

ARAÚJO, W. F.; COSTA, S. A. A.; SANTOS, A. E. dos. Comparação entre métodos de estimativa da evapotranspiração de referência (ETo) para Boa Vista - RR. Revista Caatinga, Mossoró, v. 20, n. 04, p. 84-88, 2007.

BLANEY, H. F.; CRIDDLE, W. O. Determining water requirements in irrigated areas from climatological and irrigation data. Washington: USDA Soil Conservation Service, 1950. 48p.

BORGES, A. C.; MENDIONDO, E. M. Comparação entre equações empíricas para estimativa da evapotranspiração de referência na Bacia do Rio Jacupiranga. Revista Brasileira de Engenharia Agrícola e Ambiental, Campina Grande, v. 11, n. 03, p. 293-300, 2007.

CAMARGO, A. P. Balanço hídrico no Estado de São Paulo. Campinas: IAC, 1971. $28 \mathrm{p}$.

CAMARGO, A. P.; SENTELHAS, P. C. Avaliação do desempenho de diferentes métodos de estimativa da evapotranspiração potencial no estado de São Paulo, Brasil. Revista Brasileira de Agrometeorologia, Santa Maria, v. 05, p. 89-97, 1997.

CAMARGO, A. P.; CAMARGO, M. B. P. de. Uma revisão analítica da evapotranspiração potencial. Bragantia, Campinas, v. 59, n. 02, p. 125-137, 2000.

CAVALCANTE JR., E. G.; OLIVEIRA, A. D.; ALMEIDA, B. M.; ESPÍNOLA SOBRINHO, J. Métodos de estimativa da evapotranspiração de referência para 
as condições do semiárido Nordestino. Semina, Londrina, v. 32, p. 1699-1708, 2011.

CONCEIÇÃO, M. A. F.; MANDELLI, F. Comparação entre métodos de estimativa da evapotranspiração de referência em Bento Gonçalves, RS. Revista Brasileira de Agrometeorologia, v. 13, n. 02, p. 123-127, 2005.

CUNHA, F. F. da.; MAGALHÃES, F. F.; CASTRO, M. A. de. Métodos para estimativa da evapotranspiração de referência para Chapadão do Sul - MS. Revista Engenharia na Agricultura, Viçosa, v. 21, n. 02, p. 159-172, 2013 a.

CUNHA, P. C. R.; NASCIMENTO, J. L.; SILVEIRA, P. M.; ALVES JR., J. Eficiência de métodos para o cálculo de coeficientes do tanque classe $A$ na estimativa da evapotranspiração de referência. Pesquisa Agropecuária Tropical, Goiânia, v. 43, n. 2, p. 114-122, 2013b.

DEUS, B. V. de.; ZEILHOFER, P.; ARAÚJO, G. C.; SANTOS, A. S. L. Interpolação pluviométrica na Bacia do Alto e Médio Rio Teles Pires: uma análise de séries históricas e interpoladores. In: SIMPÓSIO BRASILEIRO DE CIÊNCIAS GEODÉSICAS E TECNOLOGIAS DA GEOINFORMAÇÃO, 3., 2010, Recife. Anais... Recife - PE, 2010. p. 1-7.

DINIZ, A. F.; SANTOS, R. L.; SANTO, S. M. Avaliação dos riscos de seca para o município de Feira de Santana-BA associado à influência do El-Niño no semiárido do nordeste brasileiro. +Geografia's, Feira de Santana, n. 01, p. 18-24, 2008.

DOORENBOS, J.; PRUITT, W. O. Guidelines for predicting crop water requirement. FAO - Irrigation and Drainage, 1977, n. 24. 156 p.

FERREIRA FILHO, D. F.; BEZERRA, P. E. S.; SILVA, M. de. N. A. da.; RODRIGUES, R. S. S.; FIGUEIREDO, N. de. M. Aplicação de técnicas de interpolação para espacialização de chuvas da região hidrográfica Calha Norte, Pará. Revista Brasileira de Climatologia, Ano 15, v. 24, p. 277-299, 2019.

GOMES, D. J. C.; LIMA, A. M. M. de.; FERREIRA, N. S.; SERRÃO, E. A. O. Variabilidade espaço-temporal da precipitação: bacia hidrográfica do Rio Madeira. Revista Geografia Acadêmica, v. 13, n. 01, p. 90-104, 2019.

HARGREAVES, G. H.; SAMANI, Z. A. Reference crop evapotranspiration from temperature. Applied Engineering in Agriculture, v. 01, n. 02, p. 96-99, 1985.

HAMON, W. R. Estimating potential evapotranspiration. Journal of Hydraulics Division, ASCE, v. 87, n. 3, p. 107-120, 1961.

INSTITUTO BRASILEIRO DE GEOGRAFIA E ESTATÍSTICA (IBGE). Brasil em síntese. Disponível em: cidades.ibge.gov.br/brasil/ba/feira-desantana/panorama. Acesso em: 11 mai 2019.

INSTITUTO DA ÁGUA. Programa Nacional para o uso eficiente da água. Versão preliminar. Ministério do Ambiente e do Ordenamento do território, Lisboa, 2001. 214 p.

KHARRUFA, N. S. Simplified equation for evapotranspiration in arid regions. Beiträge zur Hydrologie, Kirchzarten, Sonderheft 5.1, p. 39-47, 1985.

LANDIM, P. M. B. Análise estatística de dados geológicos multivariados. Rio Claro, SP: UNESP, 2000. 120 p. 
LINACRE, E. T. A simple formula for estimating evaporation rates in various climates, using temperature data alone. Agricultural Meteorology, Amsterdam, v. 18, n. 06, p. 409-424, 1977.

MEDEIROS, A. T. Estimativa da evapotranspiração de referência a partir da equação de Penman-Monteith, de medidas lisimétricas e de equações empíricas, em Paraipaba, CE. Piracicaba, 2002. 103 p. Tese (Doutorado) - Escola Superior de Agricultura "Luiz de Queiroz" da Universidade de São Paulo.

MENDONÇA, E. A.; DANTAS, R. T. Estimativa da evapotranspiração de referência no município de Capim, PB. Revista Brasileira de Engenharia Agrícola e Ambiental, Campina Grande, v. 14, n. 02, p. 196-202, 2010.

MOURA, A. R. C. et al. Evapotranspiração de referência baseada em métodos empíricos em bacia experimental no estado de Pernambuco - Brasil. Revista Brasileira de Meteorologia, v. 28, n. 02, p. 181-191, 2013.

PENMAN, H. L. Natural evapotranspiration from open water, bare soil and grass. The Royal Society of London, Série A, 193, p. 120-146, 1948.

PEREIRA, A. R., VILLA NOVA, N. A., SEDIYAMA, G. C. Evapo(transpi)ração. Piracicaba: FEALQ, 1997. 183p.

PEREIRA, D. R.; YANAGI, S. N. M.; MELLO, C. R.; SILVA, A. M.; SILVA, L. A. Desempenho de métodos de estimativa da evapotranspiração de referência para a região da Serra da Mantiqueira, MG. Ciência Rural, Santa Maria, v. 39, n. 09, p. 2488-2493, 2009.

POKORNY, J. Evapotranspiration. In: FAITH, B. D. Encyclopedia of Ecology. Elsevier, 2a Ed. v. 02, p. 292-303, 2019.

PRIMO, D. F.; TREVISAN, D. P.; DUARTE, R. T. Análise comparativa de métodos de estimativa de evapotranspiração para fins agrícolas na região de São Carlos SP. Revista Brasileira de Iniciação Científica, Itapetininga, v. 06, n. 01, p. 1332, 2019.

SANTOS, R. A. dos.; MARTINS, D. L.; SANTOS, R. L. Balanço hídrico e classificação climática de Thornthwaite em Feira de Santana (BA). In: SIMPÓSIO BRASILEIRO DE CLIMATOGIA GEOGRÁFICA, 7., 2016, Goiânia. Anais... Goiânia: UFG, 2016. p. 1889-1900.

SANTOS, R. L.; ANDRADE, H. O. de. Avaliação quantitativa do conforto térmico de uma cidade em área de transição climática: Feira de Santana-Bahia, Brasil. Revista de Geografia Norte Grande, n. 40, p. 77-84, 2008.

SANTOS, W. de. O. et al. Métodos de estimativa da evapotranspiração de referência (ETo) para a região de Mossoró-RN. Revista Verde de Agroecologia e Desenvolvimento Sustentável, v. 05, n. 05, p. 210-221, 2010.

SOARES, W. A. et al. Sensibilidade do método de Linacre na estimativa da evapotranspiração. In: ENCONTRO REGIONAL DE MATEMÁTICA APLICADA E COMPUTACIONAL, 8., Natal. Anais... UFRN, 2008. 3 p.

SOUZA, J. L. de. et al. Evapotranspiração de referência estimada pelo Método de Penman-Monteith FAO-56 em função das simulações do modelo atmosférico de Mesoescala WRF - Weather Research and Forecasting. Revista Irriga, Botucatu, v. 20, n. 04, p. 762-775, 2015. 
SYPERRECK, V. L. G. et al. Avaliação de desempenho de métodos para estimativas de evapotranspiração de referência para a região de Palotina, Estado do Paraná. Acta Sci. Agron., Maringá, v. 30, p. 603-609, 2008.

TAGLIAFERRE, C.; SILVA, R.; ROCHA, F.; SANTOS, L. Estudo comparativo de diferentes metodologias para determinação da evapotranspiração de referência em Eunápolis - BA. Revista Caatinga, v. 23, n. 01, p. 103-111, 2010.

THORNTHWAITE, C. W. An approach toward a rational classification of climate. Geographical Review, New York, v. 38, n. 01, p. 55-94, 1948.

WILLMOTT, C. J. On the validation of models. Physical Geography, v. 02, p. 184194, 1981. 